\title{
Monitoramento de seca meteorológica com dados TRMM para uma região produtora de grãos do Brasil
}

Dentre todos os desastres naturais, a seca caracteriza-se como um dos mais complexo e pouco entendido. Seus efeitos impactam várias áreas da sociedade, como agropecuária, indústria, saúde, distribuição de água e geração de energia. Os índices de seca utilizados para monitorar, identificar e quantificar a anomalia de precipitação tem como principal limitação a falta de dados representativos da área de ocorrência. As medições de variáveis hidro meteorológicas por satélites oferecem uma boa alternativa na falta de dados de superfície. O objetivo do trabalho foi avaliar se o uso do produto 3B43 V7 da missão Tropical Rainfall Measuring Mission (TRMM) multi-satellite Precipitation Analysis (TMPA) é eficaz na geração da precipitação mensal e de mapas de seca a partir do Índice de Precipitação Padronizado mensal na região médio norte de Mato Grosso. Os dados foram comparados a uma base dados de superfície no período de 1998 a 2017 . A validação dos mapas de seca foi feita com base na seca que ocorreu durante a safra de 2015/16. Os resultados indicaram que o produto 3B43 V7superestima a precipitação, mas pode ser utilizado na ausência de dados de superfície, uma vez que o valor do coeficiente Nash-Sutcliffe (ENS) foi de 0,75 e do índice de concordância de Willmott (d) foi 0,93. O SPI estimado correspondeu ao observado, apresentando ENS e índice d iguais a 0,63 e 0,92, respectivamente. Os mapas de seca confirmaram a situação relatada nos boletins do Instituto Mato-grossense de Economia Agropecuária que indicaram diminuição da produtividade em decorrência da falta de chuva nos períodos críticos das culturas da soja e do milho.

Palavras-chave: TRMM; Seca; Monitoramento Agrícola; Precipitação.

\section{Meteorological drought monitoring with TRMM data for a grain producing region in Brazil}

\begin{abstract}
Drought is characterized as one of the most complex and poorly understood of all natural disasters, impacting several areas of society, such as agriculture, industry, health, water distribution and energy generation. The main limitation of drought indexes used to monitor, identify and quantify precipitation anomaly is the lack of representative data of the area of occurrence. Measurements of hydro meteorological variables by satellites provide a good alternative in the absence of surface data. Thus, the present study intends to evaluate whether the use of the 3B43 V7 product of the Tropical Rainfall Measuring Mission (TRMM) multi-satellite Precipitation Analysis (TMPA) is effective in generating monthly precipitation and drought maps from the monthly Standardized Precipitation Index in the northern region of Mato Grosso. Satellite-based data has been compared to rain gauge-based observation for the period between 1998 and 2017 . Reliability of drought maps has been verified by evaluating the great drought that occurred during the 2015/16 harvest period. Results show product 3B43 V7 overestimates precipitation, although it can be used in the absence of surface data, since Nash-Sutcliffe coefficient (ENS) value was 0.75 and the Willmott concordance index (d) was 0.93 . The correlation between SPI 3B43 V7 and SPI surface-based observation has been validated by ENS and d, whose values were 0.63 and 0.92 , respectively. The drought maps confirmed the conditions reported in the bulletins of Instituto Mato-grossense de Economia Agropecuária (IMEA), which showed crop failure due to the lack of rain throughout critical stages of soybean and corn crops.
\end{abstract}

Keywords: TRMM; Drought; Agricultural Monitoring; Rainfall.

Topic: Meteorologia, Climatologia e Mudanças Climáticas

Reviewed anonymously in the process of blind peer.
Received: 03/02/2021 Approved: $26 / 02 / 2021$
Marionei Fomaca de Sousa Junior (iD

Instituto Nacional de Pesquisas Espaciais, Brasil http://lattes.cnpq.br/7771542353189747 http://orcid.org/0000-0003-2048-6506

mariofomacajr@gmail.com

\section{Eduardo Morgan Uliana (iD)}

Universidade Federal de Mato Grosso, Brasil http://lattes.cnpq.br/2355209690082964 http://orcid.org/0000-0003-2107-4634 morganuliana@gmail.com

Mairon Anderson Cordeiro Correa de Carvalho Universidade Federal de Mato Grosso, Brasil http://lattes.cnpq.br/8953577790479587 http://orcid.org/0000-0001-5785-5841 maironcdecarvalho@gmail.com

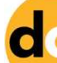

DOI: 10.6008/CBPC2179-6858.2021.002.0024

\author{
Múcio André dos Santos Alves Mendes (iD \\ Universidade Federal de Mato Grosso, Brasil \\ http://lattes.cnpq.br/5712401372315694 \\ http://orcid.org/0000-0002-8051-7805 \\ mucioandre@gmail.com \\ Luana Lisboa (iD) \\ Universidade Federal de Viçosa, Brasil \\ http://lattes.cnpq.br/8593033643157795 \\ http://orcid.org/0000-0002-7902-6072 \\ luana.lisboa@cprm.gov.br
}

\section{Referencing this:}

SOUSA JUNIOR, M. F.; ULIANA, E. M.; CARVALHO, M. A. C. C.; MENDES, M. A. S. A.; LISBOA, L.. Monitoramento de seca meteorológica com dados TRMM para uma região produtora de grãos do Brasil. Revista Ibero Americana de Ciências Ambientais, v.12, n.2, p.249-264, 2021. DOI: http://doi.org/10.6008/CBPC2179-6858.2021.002.0024 


\section{INTRODUÇÃO}

Dentre todos os desastres naturais, a seca caracteriza-se como um dos mais complexo e pouco entendido. Seus efeitos impactam várias áreas da sociedade, como agropecuária, indústria, saúde, distribuição de água e geração de energia, sendo de grande importância para o gerenciamento dos recursos hídricos (HAGMAN, 1984; PANU et al., 2002; ULIANA et al., 2015).

A definição de seca ainda é muito debatida entre os pesquisadores. Sabe-se que é uma característica normal do clima e que sua ocorrência é inevitável, mas não há um consenso na comunidade científica sobre suas características. Os impactos da seca se estendem por grandes áreas, podendo se acumular num longo período de tempo e durar anos após o término do evento (WILHITE, 2000).

Wilhite (2000) e Riebsame et al. (1991) afirmam que os prejuízos causados pela seca vêm aumentando substancialmente ao longo do tempo. Embora seja um fenômeno natural, Hagman (1984) destaca a influência do homem nesse processo por meio da degradação ambiental, afetando a frequência, severidade e efeitos da seca.

A seca é um desastre natural crônico-silencioso que causa impactos econômicos negativos a milhares de pessoas. A extensão e frequência desses eventos prejudicam diversas atividades econômicas nos setores da agropecuária, indústria, serviços e as comunidades em geral (BURITI et al., 2018). Um dos principais setores afetados é a agricultura, caracterizada como a principal provedora de alimentos e matérias-primas para energia, fornecendo benefícios diretos e indiretos para a sociedade (FORMAGGIO et al., 2017).

De acordo com o Atlas Brasileiro de Desastres Naturais (CEPED, 2013), de 1991 a 2012 foram registradas 39.837 ocorrências de desastres no Brasil, sendo que 48\% desse total (19.517 registros) foram eventos de seca e estiagem.

Conforme a Companhia Nacional de Abastecimento (CONAB, 2019), a produção brasileira de grãos na safra 2018/19 foi de 238,9 milhões de toneladas, com área plantada de 62,9 milhões de hectares. Somente no estado de Mato Grosso a produção foi estimada em 66,4 milhões de toneladas.

Dentre as diversas classificações de seca, destacam-se quatro: meteorológica, agrícola, hidrológica e socioeconômica. A seca meteorológica caracteriza-se pelo déficit de chuva em um determinado período. A seca agrícola está relacionada à disponibilidade de água no solo para o sistema radicular das plantas. A seca hidrológica reflete no nível de reservatórios e rios. Por fim, a seca socioeconômica leva em consideração a disponibilidade da água para as pessoas, bem como geração de bens e serviços (DRACUP et al., 1980; SANSIGOLO, 2004).

Com o objetivo de monitorar e caracterizar a seca, pesquisadores das mais variadas áreas desenvolveram os chamados índices de seca. Estes provêm informações importantes sobre o evento de seca, como duração, intensidade, distribuição espacial e severidade, requerendo, em geral, como dados de entrada a temperatura, umidade do solo, disponibilidade hídrica e parâmetros da precipitação (BARRA et al., 2002; FORMAGGIO et al., 2017).

A disponibilidade de dados representativos da área de ocorrência é a principal limitação do uso dos 
índices de seca. Nesse sentido, medições de variáveis hidro meteorológicas baseada em produtos de satélites oferecem uma boa alternativa na estimativa de dados em regiões com baixa densidade de estações. No entanto, a acurácia desses dados é essencial para aplicações operacionais e gerenciamento dos recursos hídricos, principalmente em áreas sujeitas a secas frequentes (PAREDES-TREJO et al., 2018).

Com base nas informações apresentadas, o objetivo do trabalho foi avaliar se o produto 3B43 V7 da missão Tropical Rainfall Measuring Mission (TRMM) multi-satellite Precipitation Analysis (TMPA) é eficaz na estimativa da precipitação e na geração de mapas de seca a partir do Índice de Precipitação Padronizado mensal (SPI-1) na região médio norte de Mato Grosso.

\section{MATERIAIS E MÉTODOS}

\section{Área de estudo e séries históricas de dados}

A área de estudo deste trabalho corresponde à região médio norte do Estado de Mato Grosso (Figura 1), Brasil, com área de aproximadamente $112.800 \mathrm{Km}^{2}$, englobando 18 municípios, sendo eles: Cláudia; Feliz Natal; Ipiranga do Norte; Itanhangá; Lucas do Rio Verde; Nova Maringá; Nova Mutum; Nova Ubiratã; Santa Carmem; Santa Rita do Trivelato; São José do Rio Claro; Sinop; Sorriso; Tapurah; União do Sul, Vera, Porto dos Gaúchos e Tabaporã. Ressaltando que esses dois últimos municípios fazem parte da região noroeste e serão incluídos no estudo devido sua influência na produção de grãos.

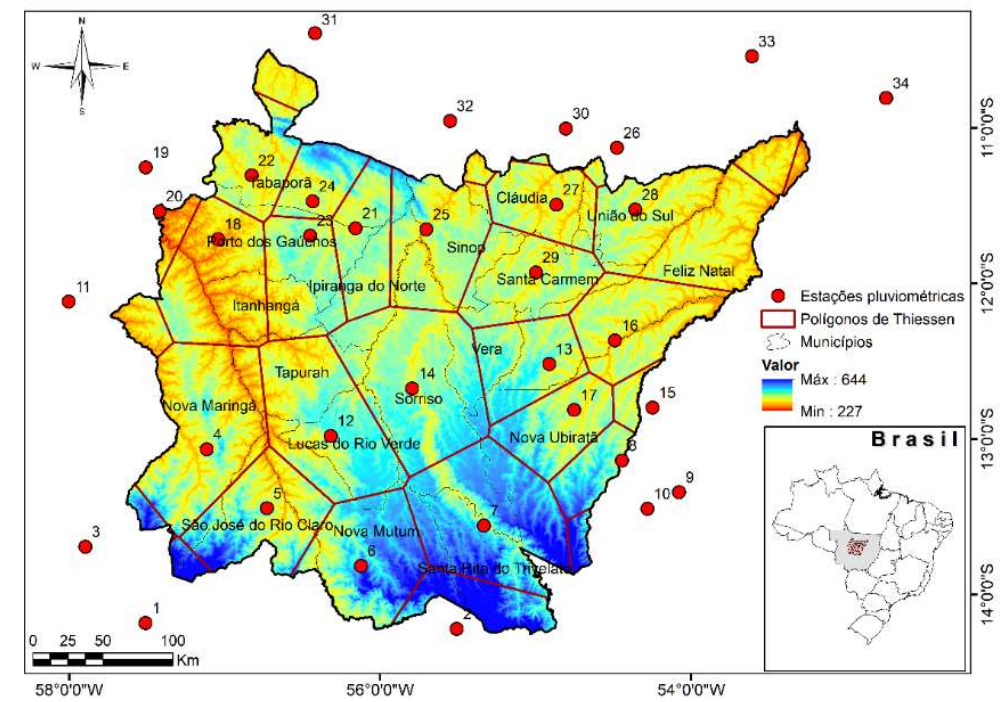

Figura 1: Localização das estações pluviométricas utilizadas no estudo.

Trata-se de uma região que se destaca na produção de grãos, cuja safra 2019/2020 de soja e milho, estimada pelo Instituto Mato-grossense de Economia Agropecuária - IMEA (2020a; 2020b), foi de 11.867 e 15.079 mil toneladas, respectivamente. Esses números correspondem a aproximadamente $34 \%$ e $45 \%$ da produção de soja e milho de todo o Estado de Mato Grosso na safra 2019/2020. A maior parte da produção de grãos da região acontece no sequeiro, o que a torna altamente vulnerável às secas.

A macrorregião média norte é caracterizada com Clima Tropical de Savana (Aw), apresentando temperaturas médias superiores a $18{ }^{\circ} \mathrm{C}$ em todos os meses do ano. Com ocorrência da estação seca nos meses de maio a setembro (outono/ inverno) e a chuvosa nos meses de outubro a abril (primavera/ verão). 
Nas regiões norte, a precipitação média anual ultrapassa a média estadual (1200 a $2000 \mathrm{~mm}$ ), podendo chegar a 2750 mm (NIMER et al., 1989; SOUZA et al., 2013; TANAKA et al., 2016).

Neste trabalho foram analisados apenas os meses do período chuvoso (meses de setembro a abril). No cerrado brasileiro a atividade agrícola concentra-se no período chuvoso (normalmente entre outubro e março), pois nessa época ocorrem de 80 a 90\% do total anual de precipitação. Além disso, o vazio sanitário da soja, principal cultura da região inicia-se em 15 de junho e vai até 15 de setembro. O período chuvoso está sujeito ainda a ocorrência de regimes de seca e estiagens, chamados de veranicos. A ocorrência desses períodos sem chuva pode causar sérios danos à agricultura, principalmente se ocorrer em períodos críticos do ciclo de desenvolvimento das culturas (ASSAD et al., 1994).

As raras precipitações que ocorrem na estação seca são, na maioria das vezes, em decorrência de encontros de massas de ar (chuvas frontais) devido a passagem de frentes polares trazidas do Sul pelo anticiclone polar (TARIFFA et al., 2006). Rosa et al. (2007) destacam ainda que a ocorrência de chuvas no período seco pode ser causada por influência do El Niño, no qual os sistemas frontais podem ficar mais ativos.

A base de dados de precipitação estimada a partir satélite foi obtida da missão Tropical Rainfall Measuring Mission (TRMM) Multisatellite Precipitation Analysis (TMPA), disponibilizada gratuitamente no site Giovanni (GES-DISC Interactive Online Visualization and Analysis Infrastructure) ${ }^{1}$. Os dados TMPA são disponibilizados na forma de produtos. Neste estudo foi utilizado o produto 3B43 V7 (1998-2017), gerado a partir da combinação de dados gerados pelo satélite TRMM e outros satélites, corrigidos a partir de estações em superfície, possuindo resolução espacial de $0,25^{\circ} \times 0,25^{\circ}$ e escala mensal. Os dados de estações pluviométricas, usados para validar o uso dos dados de sensoriamento remoto, foram obtidos no site da Agência Nacional de Águas (ANA), via Hidroweb².

Para o cálculo do Índice de Precipitação Padronizada (SPI), todas as falhas entre 1998-2017 nos dados referentes às observações de superfície foram preenchidas, após validação estatística, por dados dos produtos 3B43 V7 do TRMM Multisatellite Precipitation Analysis (TMPA), com objetivo de aumentar a quantidade de dados utilizados na obtenção dos parâmetros estatísticos do cálculo do SPI. Wu et al. (2005) afirmam que quanto maior a série de dados utilizada no cálculo do SPI mais confiáveis serão os valores do SPI. Desse modo, a base de dados observados para a validação do SPI foi composta por dados de precipitação observada em superfície e por dados baseados em satélite durante o período de 1998 a 2017. Das 34 estações utilizadas no estudo, $44,1 \%$ apresentaram 20 anos de dados, 52,9\% apresentaram mais que 30 anos de dados e $3 \%$ entre 20 e 30 anos de dados.

A localização das estações pode ser observada na Figura 1. Na Tabela 1 estão apresentadas as informações referentes às estações pluviométricas que foram utilizadas no estudo: ID, código, nome, latitude e longitude.

\footnotetext{
${ }^{1}$ https://giovanni.gsfc.nasa.gov/giovanni/

2 http://www.snirh.gov.br/hidroweb/
} 
Tabela 1: Informações das estações pluviométricas utilizadas no estudo.

\begin{tabular}{|c|c|c|c|c|c|}
\hline ID & Código & Nome & Município & Lat $\left({ }^{\circ}\right)$ & Long $\left({ }^{\circ}\right)$ \\
\hline 1 & 1457003 & Deciolândia & Diamantino & $-14,18$ & $-57,51$ \\
\hline 2 & 1455009 & Fazenda Rio Novo & Nobres & $-14,22$ & $-55,51$ \\
\hline 3 & 1357001 & Campo Novo Do Parecis & C. N. Parecis & $-13,69$ & $-57,89$ \\
\hline 4 & 1357000 & Nova Maringá & Nova Maringá & $-13,03$ & $-57,09$ \\
\hline 5 & 1356004 & São José Do Rio Claro & S. J. Rio Claro & $-13,45$ & $-56,73$ \\
\hline 6 & 1356002 & Nova Mutum & Nova Mutum & $-13,82$ & $-56,12$ \\
\hline 7 & 1355001 & Porto Roncador & S. R. Trivelato & $-13,56$ & $-55,33$ \\
\hline 8 & 1354002 & Fazenda Itaguaçu & Nova Ubiratã & $-13,14$ & $-54,44$ \\
\hline 9 & 1354001 & Agropecuária Malp & Paranatinga & $-13,34$ & $-54,08$ \\
\hline 10 & 1354000 & Fazenda Agrochapada & Paranatinga & $-13,45$ & $-54,28$ \\
\hline 11 & 1257000 & Brasnorte & Brasnorte & $-12,12$ & $-58,00$ \\
\hline 12 & 1256002 & Fazenda Divisão & L. Rio Verde & $-12,98$ & $-56,18$ \\
\hline 13 & 1255002 & Núcleo Colonial Rio Ferro & Feliz Natal & $-12,52$ & $-54,91$ \\
\hline 14 & 1255001 & Teles Pires & Sorriso & $-12,68$ & $-55,79$ \\
\hline 15 & 1254003 & Agropecuária Três Irmãos & Paranatinga & $-12,80$ & $-54,25$ \\
\hline 16 & 1254002 & Consul & Feliz Natal & $-12,37$ & $-54,49$ \\
\hline 17 & 1254001 & Agrovensa & Nova Ubiratã & $-12,81$ & $-54,75$ \\
\hline 18 & 1157002 & Olho D'água & P. dos Gaúchos & $-11,77$ & $-57,04$ \\
\hline 19 & 1157001 & Juara & Juara & $-11,25$ & $-57,51$ \\
\hline 20 & 1157000 & Porto Dos Gaúchos & P. dos Gaúchos & $-11,54$ & $-57,42$ \\
\hline 21 & 1156003 & Nova Americana & P. dos Gaúchos & $-11,64$ & $-56,16$ \\
\hline 22 & 1156002 & Tabaporã & Tabaporã & $-11,30$ & $-56,83$ \\
\hline 23 & 1156001 & Sinop (Faz. S. Verde) & P. dos Gaúchos & $-11,69$ & $-56,45$ \\
\hline 24 & 1156000 & Fazenda Itaúba & Tabaporã & $-11,47$ & $-56,43$ \\
\hline 25 & 1155000 & Cachoeirão & Sinop & $-11,65$ & $-55,70$ \\
\hline 26 & 1154005 & Riacho De Deus & Marcelândia & $-11,13$ & $-54,48$ \\
\hline 27 & 1154004 & Cláudia & Cláudia & $-11,49$ & $-54,87$ \\
\hline 28 & 1154002 & Fazenda Rio Negro & União do Sul & $-11,52$ & $-54,36$ \\
\hline 29 & 1154001 & Santa Felicidade & Santa Carmem & $-11,93$ & $-55,00$ \\
\hline 30 & 1154000 & Rancho De Deus & Marcelândia & $-11,00$ & $-54,81$ \\
\hline 31 & 1056001 & Estância Buriti & A. Floresta & $-10,40$ & $-56,42$ \\
\hline 32 & 1055003 & Fazenda Tratex & Colíder & $-10,96$ & $-55,55$ \\
\hline 33 & 1053001 & Fazenda Santa Emília & Marcelândia & $-10,54$ & $-53,61$ \\
\hline 34 & 1052000 & Vila São José do Xingu & São José do Xingu & $-10,81$ & $-52,75$ \\
\hline
\end{tabular}

\section{Índice de precipitação padronizado (SPI)}

O Índice de Precipitação Padronizado (SPI) foi calculado a partir de funções de densidade de probabilidade ajustadas às séries históricas de precipitação utilizando diferentes escalas de tempo. Após o ajuste da distribuição estatística, obteve-se a probabilidade de certa quantidade de precipitação observada e obtém-se o SPI por meio da distribuição inversa

Conforme Siqueira et al. (2015), o SPI vem sendo utilizado em vários estudos, pois é capaz de quantificar o déficit ou excesso de precipitação em diferentes escalas temporais, permitindo comparações entre regiões com características climáticas diferentes, requerendo para tal, apenas os dados pluviométricos.

Os dados observados foram ajustados na escala mensal (SPI-1) e a probabilidade acumulada de ocorrência de precipitação foi determinada pela distribuição gama incompleta definida pela seguinte função densidade de probabilidade:

$$
G(x)=\frac{x^{y-1} e^{\frac{x}{\beta}}}{\beta^{y} \Gamma(y)}
$$

Em que: $x$ é a variável aleatória; $\beta>0$ é o parâmetro de escala; $y>0$ é o parâmetro de forma; $\Gamma$ é a função gama. A função gama $\Gamma(y)$ é dada pela equação:

$$
\Gamma(y)=\int_{0}^{\infty} x^{y-1} e^{-x} d x, y>0
$$


A probabilidade acumulada de ocorrência de cada valor de precipitação foi calculada pela equação:

$$
g(x)=\int_{0}^{x} \frac{x^{y-1} e^{\frac{x}{\beta}}}{\beta^{y} \Gamma(y)} d x
$$

Os parâmetros de escala $(\beta)$ e de forma $(y)$, da função densidade de probabilidade gama foram estimados com o método da máxima verossimilhança.

A função gama incompleta não admite valores de $x=0$. Como a amostra pode conter valores nulos, a probabilidade cumulativa foi corrigida por meio da Equação 4:

$$
H(x)=q+(1-q) g(x)
$$

Onde:

$$
\mathrm{q}=\frac{\mathrm{m}}{\mathrm{n}}
$$

Sendo: q é a probabilidade de ocorrência de precipitação zero; $m$ é o número de observações com chuva igual a zero no período considerado; $n$ é o número total de valores na amostra.

$\mathrm{O}$ valor $\mathrm{H}(\mathrm{x})$ foi transformado em uma variável normal a partir das equações desenvolvidas por Abramowitz et al. (1965), resultando no valor de SPI. A relação entres as distribuições de probabilidade gama incompleta e normal foi dada por:

$$
S P I=-\left(t-\frac{c_{0}+c_{1} t+c_{2} t^{2}}{1+d_{1} t+d_{2} t^{2}+d_{3} t^{3}}\right)
$$

Para $0<H(x) \leq 0,5$.

$$
\mathrm{SPI}=+\left(\mathrm{t}-\frac{\mathrm{c}_{0}+\mathrm{c}_{1} \mathrm{t}+\mathrm{c}_{2} \mathrm{t}^{2}}{1+\mathrm{d}_{1} \mathrm{t}+\mathrm{d}_{2} \mathrm{t}^{2}+\mathrm{d}_{3} \mathrm{t}^{3}}\right)
$$

Para $0,5<H(x) \leq 1$.

Em que:

$$
\text { Sendo: } c_{0}=2,515517 ; c_{1}=0,802853 ; c_{2}=0,010328 ; d_{1}=1,432788 ; d_{2}=0,189269 ; d_{3}=0,001308 \text {. }
$$

$t=\sqrt{\ln \left(\frac{1}{(H(x))^{2}}\right)}, \quad 0<H(x) \leq 0,5$

e

$t=\sqrt{\ln \left(\frac{1}{(1-H(x))^{2}}\right)}, 0,5<H(x) \leq 1$

Na Tabela 2 encontra-se a classificação para déficit e excesso de precipitação em função do valor do SPI. Destaca-se que o evento de seca começa quando o valor do SPI se torna negativo e termina quando se torna positivo.

Tabela 2: Classificação dos períodos de seca e umidade do SPI, conforme Mckee et al. (1993).

\begin{tabular}{ll}
\hline Categoria & Valor SPI \\
\hline Extremamente Úmido & $\mathrm{SPI} \geq 1,5$ \\
Moderadamente Úmido & $1 \leq \mathrm{SPI}<1,5$ \\
Umidade Incipiente & $0 \leq \mathrm{SPI}<1$ \\
Seca Incipiente & $-1<\mathrm{SPI}<0$ \\
Moderadamente Seco & $-1,5<\mathrm{SPI} \leq-1$ \\
Extremamente Seco & $\mathrm{SPI} \leq-1,5$ \\
\hline
\end{tabular}

Como o SPI baseado no produto 3B43 V7 foi calculado a partir dos parâmetros $\beta$ e y obtidos a partir 
da série de dados observados, que foi composta por dados de estações em superfície e dados de satélite, foi necessário estabelecer limites de influência para cada conjunto de parâmetros, uma vez que são obtidos separadamente para cada uma das 34 séries de dados. A área de influência de uma estação pluviométrica foi calculada pelo método de Thiessen que, segundo Tucci (2004), considera a não uniformidade das estações pluviométricas dispostas no espaço, não levando em consideração o relevo da área. Definida a área de influência dos parâmetros $\beta$ e y, o SPI baseado nos dados TRMM foi calculado para cada pixel.

\section{Validação estatística}

A região média norte compreendeu 152 pixels, uma vez que a resolução espacial do produto 3B43 V7 é de $0,25^{\circ} \times 0,25^{\circ}$. Os dados de 34 estações pluviométricas disponibilizados pela Agência Nacional de Águas (ANA) foram usados para validação dos produtos 3B43 V7 na geração da precipitação mensal. A partir das coordenadas geográficas das estações pluviométricas da ANA foi possível extrair os valores de precipitação dos pixels e compará-los aos valores observados nas estações de superfície. A validação do SPITRMM foi feita com base na média mensal de todos os pixels para cada mês dos períodos chuvosos dos anos de 1998 a 2017. Para melhor visualização dos mapas de seca a partir do SPI-TRMM os pixels foram divididos por 80 e interpolados pelo método bilinear.

A validação do produto 3B43 V7 foi feita com base no erro absoluto médio (MAE), raiz do erro quadrático médio (RMSE), viés, índice de concordância de Willmott (d), coeficiente Nash-Sutcliffe (ENs) e coeficiente de correlação de Pearson ( $r$ ). Detalhes sobre a avaliação do erro podem ser consultados em Uliana et al. (2020).

\section{RESULTADOS}

\section{Validação do satélite Tropical Rainfall Measuring Mission (TRMM)}

O desempenho do satélite Tropical Rainfall Measuring Mission (TRMM) na estimativa de séries mensais de precipitação está exposto na Tabela 3. A Tabela apresenta as medidas quantitativas: erro absoluto médio (MAE), viés, índice de Willmott (d), coeficiente Nash-Sutcliffe ( $\left.E_{N S}\right)$ e coeficiente de correlação de Pearson ( $r)$.

Tabela 3: Medidas quantitativas de desempenho do satélite Tropical Rainfall Measuring Mission (TRMM) na geração de séries mensais de precipitação.

\begin{tabular}{lllllll}
\hline Estação & MAE & RMSE & viés & $\mathrm{d}$ & $\mathrm{E}_{\text {NS }}$ & $\mathrm{r}$ \\
\hline 1052000 & 51.64 & 76.76 & -18.60 & 0.92 & 0.64 & 0.94 \\
1053001 & 58.61 & 91.21 & -32.79 & 0.89 & 0.54 & 0.92 \\
1053003 & 41.51 & 62.21 & -0.70 & 0.96 & 0.84 & 0.96 \\
1056001 & 47.11 & 69.68 & -3.46 & 0.94 & 0.77 & 0.95 \\
1154000 & 53.59 & 85.02 & -12.92 & 0.92 & 0.68 & 0.93 \\
1154001 & 46.45 & 71.86 & 4.78 & 0.93 & 0.76 & 0.93 \\
1154002 & 51.60 & 80.97 & -15.72 & 0.93 & 0.70 & 0.94 \\
1154004 & 60.63 & 94.20 & 34.47 & 0.93 & 0.79 & 0.96 \\
1154005 & 43.90 & 64.78 & -17.59 & 0.95 & 0.80 & 0.96 \\
1155000 & 41.16 & 64.54 & -10.41 & 0.95 & 0.79 & 0.95 \\
1156000 & 46.47 & 69.89 & -7.94 & 0.94 & 0.78 & 0.95 \\
1156001 & 46.04 & 66.90 & -7.95 & 0.95 & 0.79 & 0.95 \\
1156002 & 52.51 & 88.10 & 12.34 & 0.91 & 0.71 & 0.93
\end{tabular}




\begin{tabular}{|c|c|c|c|c|c|c|}
\hline 1156003 & 48.72 & 80.42 & 4.88 & 0.93 & 0.76 & 0.94 \\
\hline 1157000 & 43.03 & 64.87 & -9.88 & 0.95 & 0.78 & 0.95 \\
\hline 1157001 & 43.00 & 64.29 & -1.91 & 0.95 & 0.79 & 0.95 \\
\hline 1157002 & 40.10 & 60.00 & 1.94 & 0.96 & 0.83 & 0.96 \\
\hline 1254001 & 41.72 & 66.00 & -25.03 & 0.94 & 0.72 & 0.95 \\
\hline 1254002 & 51.65 & 84.04 & -14.88 & 0.90 & 0.60 & \\
\hline 1254003 & 71.17 & 122.19 & 15.77 & 0.86 & 0.61 & \\
\hline 1255002 & 41.89 & 64.71 & -17.10 & 0.94 & 0.77 & \\
\hline 1256002 & 37.43 & 56.30 & -6.78 & 0.96 & 0.84 & \\
\hline 1257000 & 41.64 & 63.72 & -10.58 & 0.94 & 0.77 & \\
\hline 1354000 & 43.03 & 67.77 & -4.55 & 0.94 & 0.77 & \\
\hline 1354001 & 46.93 & 73.71 & -21.00 & 0.92 & 0.70 & \\
\hline 1356004 & 46.60 & 72.79 & -9.73 & 0.92 & 0.74 & 0. \\
\hline 1357000 & 51.60 & 77.38 & -9.07 & 0.92 & 0.73 & 0. \\
\hline 1357001 & 41.30 & 64.58 & -3.99 & 0.93 & 0.76 & \\
\hline 1455009 & 43.61 & 67.85 & -7.23 & 0.93 & 0.75 & \\
\hline 1457003 & 46.54 & 65.98 & -6.43 & 0.94 & 0.77 & \\
\hline Média & 46.55 & 72.27 & -7.25 & 0.93 & 0.75 & \\
\hline
\end{tabular}

MAE: erro absoluto médio (mm); RMSE: raiz do erro quadrático médio; d: índice de concordância de Willmott; e ENs: coeficiente Nash-Sutcliffe.

De acordo com a Tabela 3 a precipitação mensal do produto 3B43 V7 do satélite TRMM tende a ser maior que a precipitação observada nas estações pluviométricas, uma vez que valor do viés foi negativo na maioria das estações. Os valores mínimo e máximo do viés foram de -32,79mm (estação 1053001) e 34,47mm (estação 1154004), respectivamente.

Quando se analisa os valores médios do índice de Willmott (d) e coeficiente Nash-Sutcliffe ( $E_{N S}$ ) temse que foram iguais a 0,75 e 0,93, respectivamente. Conforme a classificação proposta por Van Liew et al. (2007), a estimativa de precipitação mensal do TRMM foi classificada como satisfatória uma vez que esteve entre $0,36<E_{N s} \leq 0,75$. Quando se analisa os valores do coeficiente de Willmott deve-se ter em mente que quanto mais próximo da igualdade melhor a concordância dos dados estimados em relação aos observados, enquanto que o valor de MAE igual a zero indica ajuste perfeito dos dados preditos em relação aos estimados. Além disso, o valor médio do $r$ foi de 0,94 , indicando também boa correlação entre os dados.

O menor desempenho foi observado na estação $1053001 \quad\left(E_{N s}=0,54\right)$ enquanto que o melhor desempenho obtido na estação 1355001 ( $\left.E_{N S}=0,84\right)$. De acordo com Cao et al. (2018), a acurácia dos dados de precipitação estimados pelo TRMM depende de vários fatores como região, estação do ano, escalas temporal e espacial, etc. Os referidos autores relataram também que a rugosidade da superfície influencia no desempenho de produtos do TRMM, obtendo resultados mais satisfatórios do produto 3B43 em áreas urbanas e de agricultura, ao contrário de regiões de floresta e água. Outro resultado obtido pelos autores foi que o produto 3B43 superestimou a precipitação em todos os casos.

Os resultados indicaram que os dados do produto 3B43 V7 do satélite Tropical Rainfall Measuring Mission (TRMM) podem ser usados na ausência de dados de estações de superfície, uma vez que representaram de forma adequada a precipitação na escala mensal na região médio norte de Mato Grosso. 


\section{Distribuição espacial da precipitação na mesorregião médio norte}

Ao analisar a distribuição espacial da precipitação média anual $(\mathrm{mm})$ na região médio norte (Figura 2) verifica-se uma tendência de aumento da precipitação à medida que se aproxima da região norte. Os menores índices pluviométricos são na região mais ao sul. A precipitação anual na região médio norte variou de 1755 mm a 2085 mm, no qual os maiores índices de chuva foram observados em Tabaporã, Porto dos Gaúchos, Itanhangá, Tapurah, Ipiranga do Norte, Cláudia, União do Sul, Sinop e Feliz Natal.

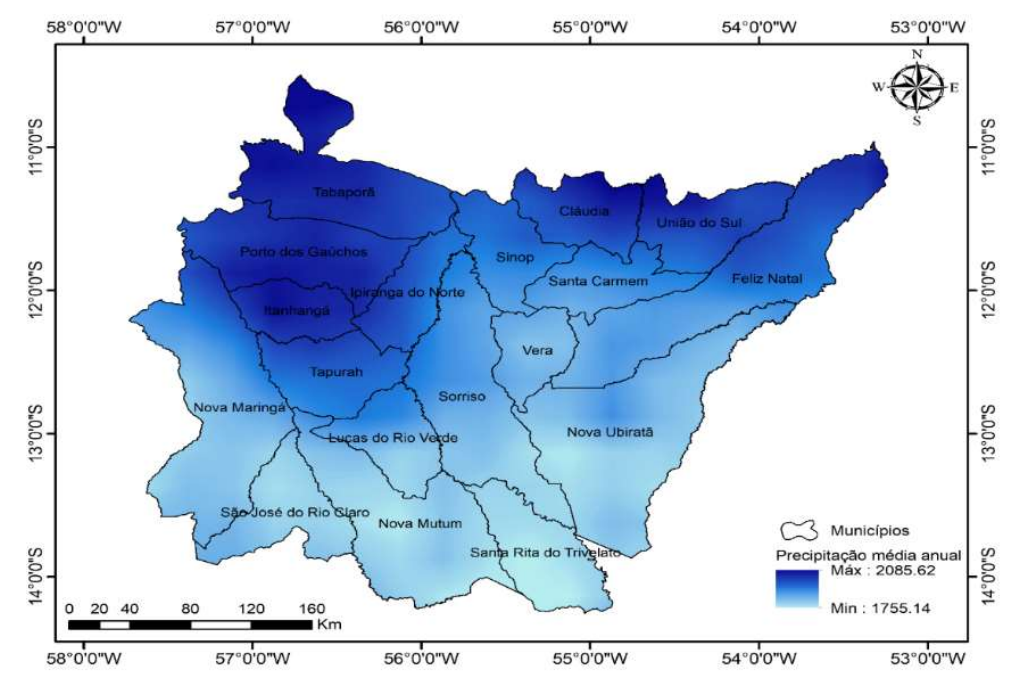

Figura 2: Distribuição espacial da precipitação média anual (TRMM) na região médio norte de Mato Grosso.

\section{Validação do SPI baseado no TRMM}

A Figura 3 apresenta o comportamento médio mensal para o período chuvoso do SPI calculado a partir da série de dados observada e a do SPI obtido por dados do TRMM.

A partir de uma análise visual nota-se uma boa concordância com do SPI-TRMM com o SPI observado com maiores dificuldades de representação dos extremos. Alguns valores de seca foram estimados com maior intensidade, como no caso da seca entre anos de 2007-2008 e 2010-2011, mas de forma geral o SPITRMM reproduz a variação temporal do SPI observado. A estimativa de eventos de seca com maior intensidade também foi relatada por Tan et al. (2017) ao utilizar o produto 3B43 na geração do SPI mensal na Malásia. Os valores do MAE e viés foram 0,27 e -0,08, respectivamente. Quanto ao valor do $E_{N S, ~} 0$ desempenho foi classificado como satisfatório $\left(E_{N s}=0,63\right)$ pela classificação de Van Liew et al. (2007). O valor do índice 'd' demonstra também uma boa concordância entre o SPI-TRMM e o SPI observado uma vez que seu valor foi igual a 0,92.

O bom desempenho do SPI mensal para a região médio norte também é observado na Figura 4. Notase boa correlação entre os dados, com uma dispersão maior nos menores valores de SPI, o que também demonstra a estimativa de maior intensidade dos eventos seca. O coeficiente de correlação de Pearson foi de 0,88. A inclinação da reta da regressão demonstra uma tendência de superestimativa do SPI.

Na figura 5 são apresentadas todas as faixas de SPI médio da região médio norte para cada mês do período de 1998 a 2017 baseado nos dados do TRMM. 


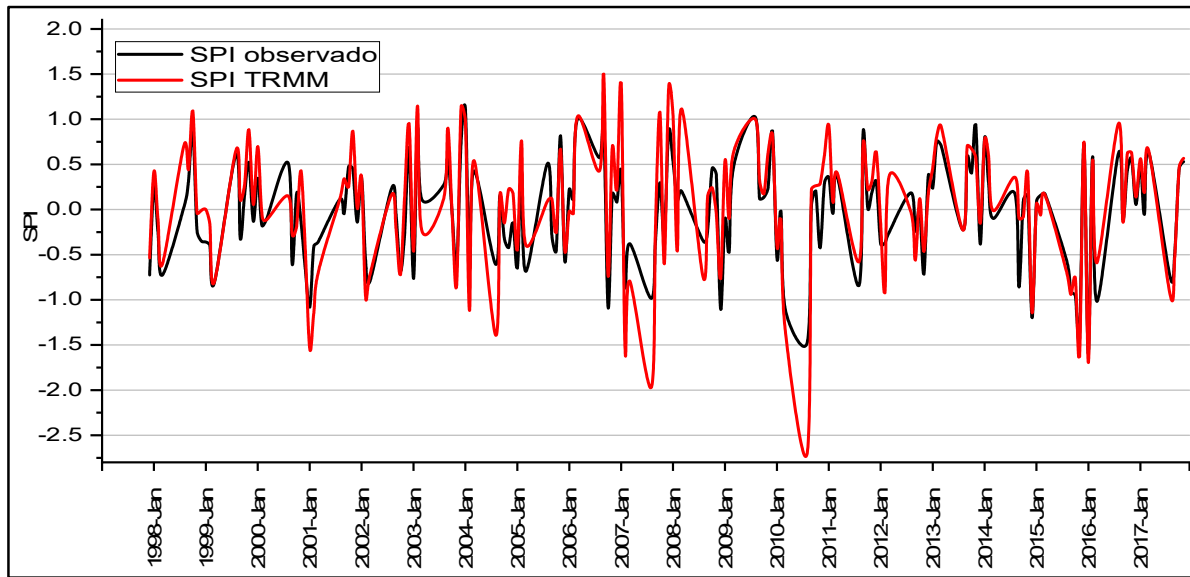

Figura 3: Comparação entre o SPI mensal calculado a partir da série de dados observados e o SPI calculado a partir de dados do produto 3B43 V7.

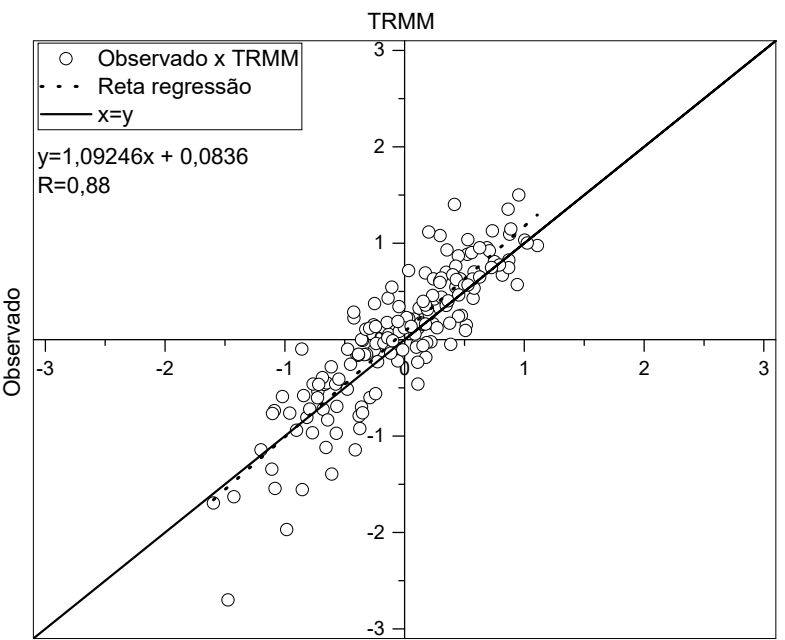

Figura 4: Comparação entre o SPI-TRMM e SPI observado na escala mensal.

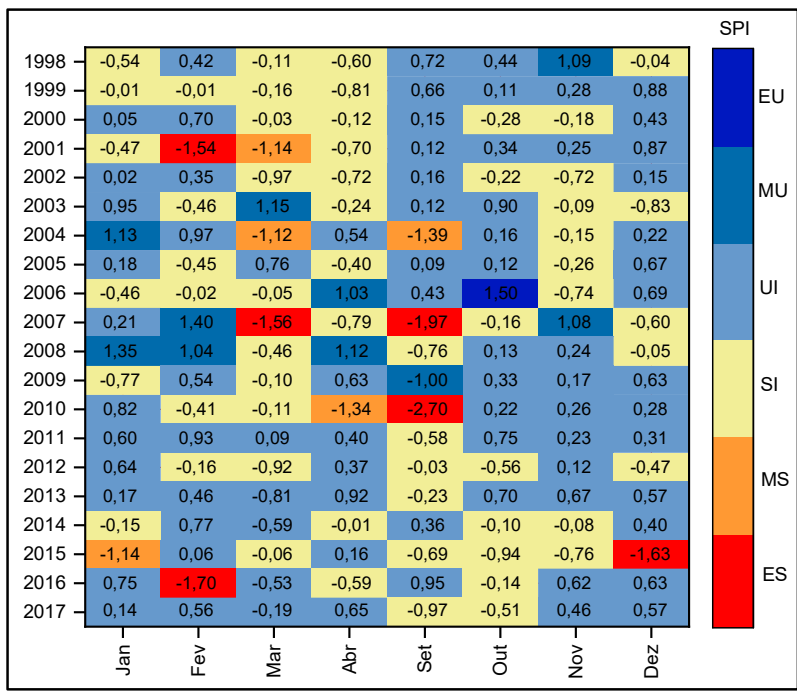

Figura 5: SPI médio mensal da região médio norte baseado em dados do TRMM. ES: extremamente seco;

MS: moderadamente seco; SI: seca incipiente; UI: umidade incipiente; MU: moderadamente úmido; EU: extremamente úmido.

\section{Estudo de caso safra 15/16}

A exemplo das consequências do estresse hídrico na agricultura, tem-se a safra 2015-2016. O Instituto Mato-grossense de Economia Agropecuária (IMEA) realizou o 1ㅇ levantamento das condições de lavouras de soja em Mato Grosso em decorrência das adversidades climáticas da região.

Conforme o IMEA (2015a) houve um atraso no início das chuvas em que apenas $38 \%$ da área destinada à cultura da soja no estado estavam semeadas no final de outubro de 2015. No entanto, na região médio norte $46,3 \%$ da área já estava semeada no mesmo período e $92,5 \%$ da área antes da primeira quinzena de novembro de 2015. Dessa forma, o ritmo de semeadura mais acelerado na região médio norte fez com que o período reprodutivo da maioria das lavouras de soja coincidisse com o mês de dezembro, principalmente o estádio de floração, tido como um período crítico para o desenvolvimento da cultura.

De acordo com o IMEA (2015b), no estudo realizado entre 16 e 18 de dezembro de 2015, a região média norte do estado estava entre as mais afetadas pela seca. Cerca de 53\% das lavouras estavam em situação ruim ou péssima com risco da produtividade ficar abaixo de 50 sc/ha. Na primeira semana de janeiro de 2016 foi realizado o segundo levantamento e constatou-se que com o aumento do volume de chuvas 
nesse mês houve uma melhora nas condições das lavouras, diminuindo a porcentagem de lavouras em condições ruins ou péssimas para 38\% (IMEA, 2016a). No terceiro levantamento (IMEA, 2016b), essa porcentagem diminuiu para $31 \%$.

Na Figura 5 observa-se que a partir de setembro inicia-se um período de seca na região médio norte. Este mês é caracterizado pelo fim do vazio sanitário e início da semeadura da soja a partir da segunda quinzena. O mês de dezembro apresentou o pico de seca, sendo caracterizado como mês extremamente seco (SPI médio da região igual a -1,63). Como já mencionado, este mês coincidiu com o início do estádio de floração da maioria das lavouras da região. Em janeiro de 2016 tem-se que realmente o volume de chuvas aumentou (SPI médio igual a 0,75 ), classificando o valor do SPI nesse mês como umidade incipiente. Neste mês parte das lavouras da região ainda estavam no período reprodutivo, o que fez com que a perda de produtividade estimada devido ao déficit hídrico no início da safra pudesse ser amenizada.

Mesmo com amenização dos danos devido ao aumento das chuvas no mês de janeiro de 2016, no final da safra 15/16 o IMEA (2016c) relatou uma queda de 3,92\% da produção total do estado em relação à safra $14 / 15$, correspondente a 1,1 milhões de toneladas, devido às condições climáticas que ocorreram durante a safra. A região média norte foi a mais afetada, com queda de produção e produtividade de 10,33\% e $11,05 \%$, respectivamente, em relação à safra anterior.

Na safra 15/16 ocorreu um atraso na colheita da soja, o que consequentemente atrasou a semeadura do milho, devido ao excesso de chuva em janeiro. No final de janeiro cerca de 5,45\% da área total destinada ao milho no estado de Mato Grosso tinham sido semeadas, deixando a maior parte da área para ser semeada em fevereiro, conforme dados do IMEA (2016d).

Com o atraso da semeadura, na região médio norte cerca de 450 mil hectares foram semeados fora da "janela ideal" (final de fevereiro), de acordo com o IMEA (2016d). Isso fez com que o período crítico da cultura do milho coincidisse com o mês de abril que apresentou pluviosidade abaixo do necessário para o desenvolvimento da planta. Nesse mês, 72,1\% das lavouras pendoaram (IMEA, 2016e).

A décima estimativa de safra realizada pelo IMEA apontou queda de 7,3 milhões de toneladas em relação à safra $14 / 15$. Somente na região médio norte houve uma queda de produtividade de $29,2 \%$ em relação à safra anterior. Essa queda fez com que, no estado, cerca de 177,7 mil hectares fossem abandonados, sendo que 39,9 mil hectares foram abandonados apenas na região médio norte (IMEA, 2016e; IMEA, 2016f).

A Figura 7 apresenta a distribuição espacial dos eventos de seca e umidade nos meses de setembro de 2015 a abril de 2016. Percebe-se o início de evento de seca no mês de setembro, com aumento da severidade até o mês de dezembro, no qual quase toda região média norte encontra-se sob extrema seca. Conforme mencionado, houve um atraso na semeadura da cultura da soja devido ao atraso do início das chuvas, se agravando ainda mais no mês dezembro, no qual ocorreu uma situação de alto estresse hídrico na cultura, coincidindo com período crítico das lavouras, principalmente na região médio norte. 


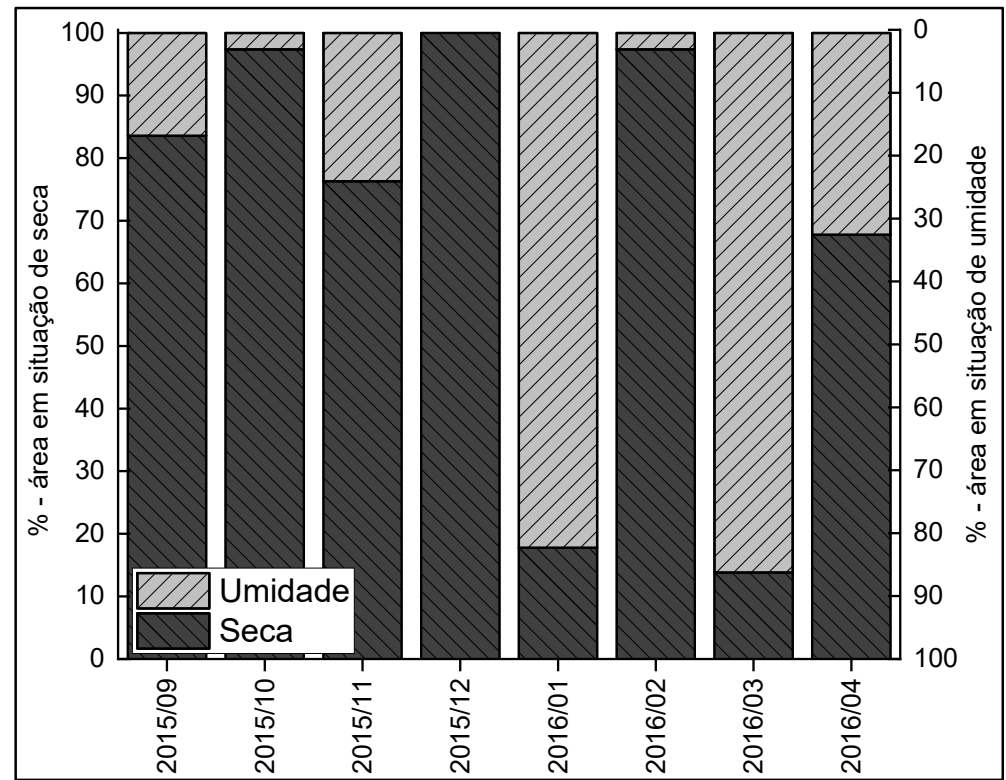

Figura 6: Porcentagem dos grids em situação de umidade e seca na região médio norte de Mato Grosso.

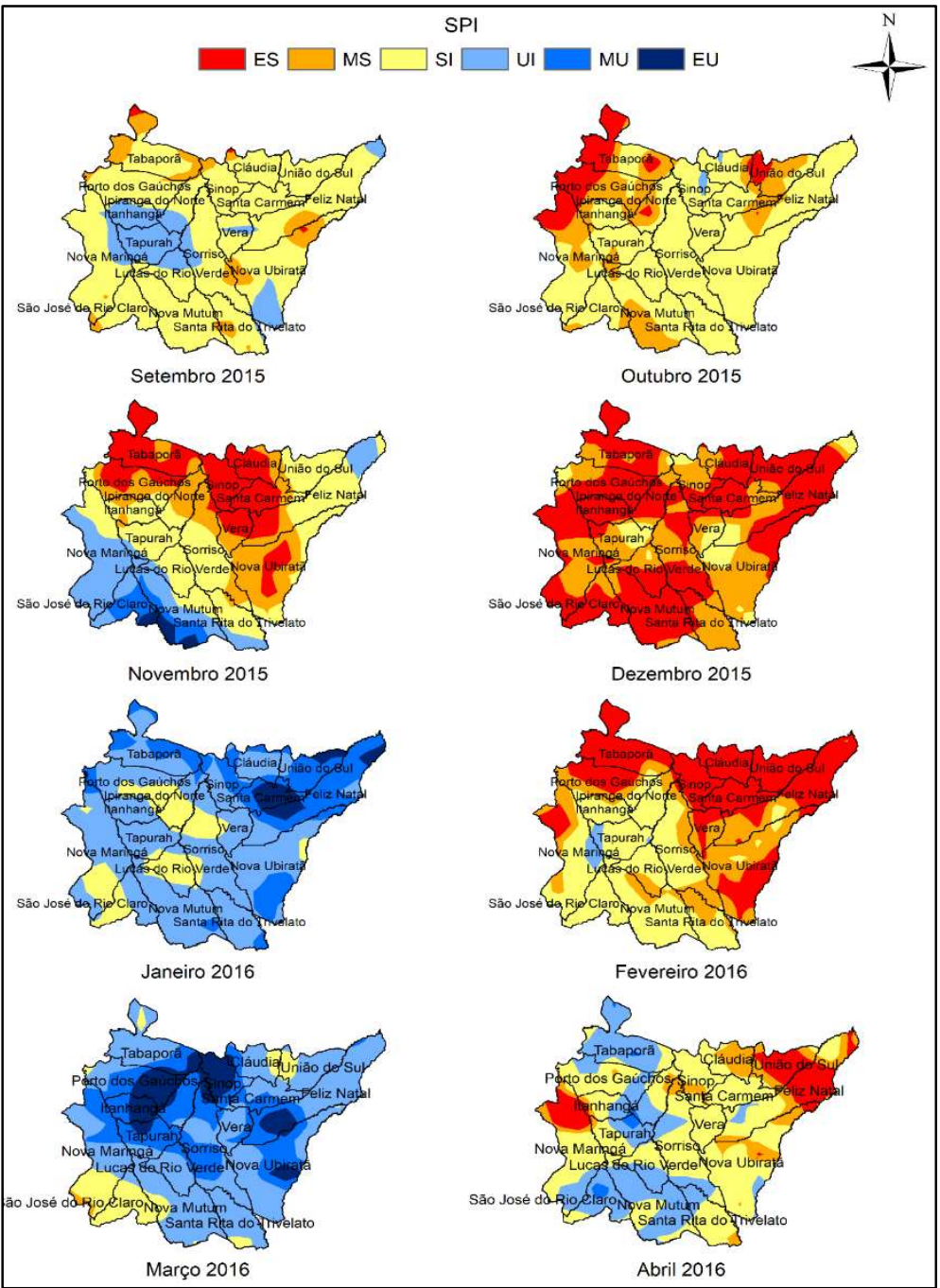

Figura 7: SPI mensal na região médio norte no período de setembro de 2015 a fevereiro de 2016 baseado em dados do TRMM. ES: extremamente seco; MS: moderadamente seco; SI: seca incipiente; UI: umidade incipiente; MU: moderadamente úmido; EU: extremamente úmido.

No mês de janeiro (Figura 7) nota-se o oposto, com áreas em estado de extrema umidade, o que explica o a recuperação de boa parte das lavouras de soja, uma vez que as mesmas ainda se encontravam em período de floração-enchimento de grãos. A umidade extrema de janeiro de 2016 também explica o 
atraso da semeadura do milho relatadas anteriormente, uma vez que o excesso de precipitação impossibilita a colheita da soja e posterior semeadura da cultura do milho. O mês de fevereiro, no entanto, assim como no mês de dezembro, encontra-se com boa parte da região médio norte sob extrema seca. Por fim, o mês de abril apresentou eventos de seca em boa parte da região, coincidindo também com o período crítico do milho o que permite explicar a queda de produtividade daquele ano.

A Figura 6 apresenta a porcentagem de área em situação de seca (SPI negativo) e umidade (SPI positivo) para os meses de setembro de 2015 a abril de 2016. No mês de setembro, outubro e novembro as porcentagens de área em estado de seca foram de, respectivamente, 83,6\%, 97,4\% e 76,3\%. Dezembro de 2015 apresentou 100\% da região em estado de seca, enquanto que fevereiro de 2016 apresentou 97,4\%. No mês de abril cerca de $67,8 \%$ da região médio norte estava em situação de seca. A situação de umidade prevaleceu nos meses de janeiro e março de 2016, com $82,2 \%$ e $86,2 \%$ da área nessa situação, respectivamente.

Isso demonstra o bom desempenho da geração dos mapas de seca da região médio norte a partir dos dados de sensoriamento remoto. Destacando a identificação dos períodos de falta e excesso de chuva, principalmente em relação aos períodos críticos mencionados das culturas da soja e do milho que influenciam diretamente no desempenho da produtividade desses commodities.

\section{DISCUSSÃO}

Diversos são os trabalhos que utilizam os dados de precipitação baseados em satélite para monitoramento da seca. Jesús et al. (2016) compararam dados de SPI baseados no satélite TRMM e em estações de superfície para monitorar eventos de seca no México. Os referidos autores obtiveram, para o SPI mensal, coeficiente de correlação de Pearson de 0,41 para regiões áridas e -0,07 para regiões de clima temperado ao analisarem o SPI gerado a partir de estações de superfície e o SPI gerado a partir de dados do TRMM. O mau desempenho do SPI baseado em dados de satélite foi associado à quantidade dos registros de precipitação a partir dos quais o SPI foi calculado e à baixa concordância entre as estimativas de precipitação entre as estações pluviométricas e os dados baseados no TRMM. Os autores também sugeriram a utilização de uma base de dados mistas entre os dados baseados em satélite e baseados em estações de superfície.

A utilização de uma base de dados formada a partir desses dois tipos de dados para obter os parâmetros estatísticos para o cálculo do SPI se mostrou eficaz numa região de clima tropical, como mostrado neste trabalho. O coeficiente de correlação de Pearson obtido aqui, entre o SPI calculado a partir de uma base mista entre dados de superfície e de satélite e o SPI calculado a partir de dados de satélite, foi de 0,88 , demonstrando boa correlação entre os dados.

No estudo realizado em uma bacia da Malásia, Tan et al. (2017) obtiveram bom desempenho do produto 3B43 na estimativa mensal de precipitação $(R=0,75)$ de 42 estações (1998-2014), no entanto afirmaram que o produto não é adequado para ser usado diretamente para a estimativa de SPI na bacia em questão, requerendo maior precisão. Daí a importância da obtenção dos parâmetros estatísticos utilizados 
no cálculo do SPI a partir de uma série de dados que mescle os dados do produto 3B43 com os dados observados em superfície, pois, em muitos casos, a série de dados observados é maior que a série de dados baseados em satélite.

Zhao et al. (2018) utilizaram o SPI baseado no TRMM para identificar tendências de seca na região de Loess Plateau, na China. Os autores identificaram áreas com riscos de seca e umidade na região, correlacionando essas alterações com as mudanças do uso do solo.

O monitoramento de seca baseado em dados de satélite e a determinação da sua acurácia em relação aos dados observados em superfície é de extrema importância na mitigação dos impactos causados por esse desastre natural, além do gerenciamento dos recursos hídricos, principalmente em setores que dependem grandemente da precipitação, como a agricultura.

O SPI baseado no produto 3B43 V7, calculado a partir dos parâmetros $\beta$ e y obtidos por uma série de dados que mesclam os dados disponíveis das estações pluviométricas com o preenchimento de falhas feitos por dados baseados em satélite, é eficaz na identificação de regiões com estresse hídrico. Os mapas de seca gerados permitem correlacionar a falta ou excesso de água com as situações encontradas nas lavouras da região, podendo explicar as quebras de safras em anos de condições climáticas extremas.

A geração dos mapas de secas também pode ser útil para auxiliar a tomada de decisão nos seguros agrícolas, uma vez que permite a identificação desses eventos extremos durante a safra. Além disso, a validação do produto 3B43 V7 na geração da precipitação mensal permite a geração de dados na ausência de estações pluviométricas, auxiliando os estudos hidrológicos da região.

Este trabalho mostra o potencial do uso de medições de variáveis hidrometeorológicas feitas a partir de dados de sensoriamento remoto na região médio norte de Mato Grosso. Embora a região estudada seja a maior produtora de grãos do Brasil, sendo esta uma atividade que depende totalmente das condições climáticas para um bom desempenho produtivo, ainda carece de dados hidrometeorológicos de qualidade e em larga escala que possam ser utilizados na tomada de decisão. Recomendam-se outros estudos que validem a nova geração de dados de precipitação gerados a partir de plataformas orbitais, como o Global Measuring Mission que possui maior resolução espacial $\left(0,1^{\circ}\right)$ e promete uma melhor estimativa de eventos de baixa intensidade. Além disso, estudos que identifiquem as tendências no comportamento das secas ajudam no entendimento dos impactos causados pela produção agropecuária na região.

Portanto, a partir das informações expostas, pode-se afirmar dados do produto 3B43 V7 do TRMM representam de forma correta os eventos de seca e umidade na região médio norte de Mato Grosso e pode ser usado na geração de mapas de seca a partir do Índice de Precipitação Padronizado (SPI).

\section{CONCLUSÕES}

O produto 3B43 V7 da missão Tropical Rainfall Measuring Mission (TRMM) multi-satellite Precipitation Analysis (TMPA) é eficaz na geração da precipitação mensal na região médio norte de Mato Grosso. É possível determinar mapas de seca mensais com base no índice de Precipitação Padronizado (SPI). O uso de dados hidrometeorológicos baseados em satélite é uma solução eficiente na ausência de dados de 
superfície, podendo auxiliar o planejamento agrícola e gerenciamento dos recursos hídricos.

\section{REFERÊNCIAS}

ABRAMOWITZ, M.; STEGUN, I. A.. Handbook of mathematical function. Dover Publications, 1965.

ASSAD, E. D.; SANO, E. E.; MASUTOMO, R.; CASTRO, L. H.; SILVA, F. A. M.. Veranicos na região dos cerrados brasileiros frequência e probabilidade de ocorrência. In: ASSAD, E. D.. Chuva nos Cerrados. Brasília: EMBRAPA, 1994. p.423.

BARRA, T. S.; COSTA, J. N. M.; RAO, T. V. R.; SEYDIAMA, G. C.; FERREIRA, W. P. M.; NETO, F. S. D.. Caracterização climatológica da severidade das secas do Estado do Ceará Brasil. Revista Brasileira de Engenharia Agrícola e Ambiental, Campina Grande, v.6, n.2, p.266-272, 2002. DOI: http://dx.doi.org/10.1590/S1415-43662002000200015

BURITI, C. O.; BARBOSA, H. A.. Um século de secas: Por que as políticas públicas não transformaram o semiárido brasileiro?. São Paulo: Chiado, 2018.

CAO, Y.; ZHANG, W.; WANG, W.. Evaluation of TRMM 3 B43 data over Yangtze River Delta of China. Scientific Reports, n.8, p.1-12, 2018. Dol: https://doi.org/10.1038/s41598-018$\underline{23603-z}$

CEPED. Centro de treinamento em São Miguel do Oeste. Atlas Brasileiro de Desastres Naturais (1991-2012). Florianópolis: UFSC, 2013.

CONAB. Companhia Nacional de Abastecimento. Acompanhamento da safra brasileira- safra 2018/19, v.6, n.9- Nono levantamento. Brasília: Ministério da Agricultura, Pecuária e Abastecimento, 2019.

DRACUP, J. A.; LEE, K. S.; PAULSON, E. G. J.. On the definition of drought. Water Resources Research, v.16, n.2, p.279-302, 1980. DOI: https://doi.org/10.1029/WR016i002p00297

FORMAGGIO, A. R.; SANCHES, I. D.. Sensoriamento remoto em agricultura. São Paulo: Oficina de Textos, 2017.

HAGMAN, G.. Prevention better than cure: report on human and environmental disasters in the Third World. Stockholm: Swedish Red Cross, 1984.

IMEA. Instituto Mato-grossense de Economia Agropecuária. 4a Estimativa da Safra de Soja - 2019/20. Cuiabá: IMEA, 2020a.

IMEA. Instituto Mato-grossense de Economia Agropecuária. 5a Estimativa da Safra de Milho - 2019/20. Cuiabá: IMEA, 2020b.

IMEA. Instituto Mato-grossense de Economia Agropecuária. $\mathbf{2}^{\circ}$ Levantamento das condições de lavouras de soja: Safra 2015/16 em Mato Grosso. Cuiabá: IMEA, 2016a.

IMEA. Instituto Mato-grossense de Economia Agropecuária. $3^{\circ}$ Levantamento das condições de lavouras de soja: Safra 2015/16 em Mato Grosso. Cuiabá: IMEA, 2016b.

IMEA. Instituto Mato-grossense de Economia Agropecuária. Boletim semanal da soja: n. 404. Cuiabá: IMEA, 2016 c.
IMEA. Instituto Mato-grossense de Economia Agropecuária. Boletim semanal do milho: n. 388. Cuiabá: IMEA, 2016d.

IMEA. Instituto Mato-grossense de Economia Agropecuária. Boletim semanal do milho. n. 398. Cuiabá: IMEA, 2016e.

IMEA. Instituto Mato-grossense de Economia Agropecuária. $1^{\circ}$ Estimativa de abandono de área da safra 15/16 de milho em Mato Grosso: n. 417. Cuiabá: IMEA, $2016 \mathrm{f}$.

IMEA. Instituto Mato-grossense de Economia Agropecuária. Boletim semanal da soja, Cuiabá: IMEA, 2015a.

IMEA. Instituto Mato-grossense de Economia Agropecuária. $1^{\circ}$ Levantamento das condições de lavouras de soja: Safra 2015/16 em Mato Grosso. IMEA. Cuiabá: IMEA, $2015 b$.

JESÚS, A.; BREÑA-NARANJO, J. A.; ACUÑA-PEDROZO, A.; YAMANAKA, V. H. A.. The use of TRMM 3442 Product for DROUGHT Monitoring in Mexico. Water, v.8, n.325, p.2-18, 2016. DOI: https://doi.org/10.3390/w8080325

MCKEE, T. B.; DOESKEN, N. J.; KLEIST, J.. The relationship of drought frequency and duration to time scales. In: CONFERENCE ON APPLIED CLIMATOLOGY, 1993. Anais. Anaheim: American Meteorological Society, 1993. p.179184.

NIMER, E.; BRANDÃO, A. M. P. M.. Balanço hídrico e clima da região dos cerrados. Rio de Janeiro: IBGE, 1989.

PANU, U. S.; SHARMA, T. C.. Challenges in drought research: some perspective and future directions. Hydrological Sciences- Journal-des Sciences Hydrologiques, v.47, n.1, p.19-30, 2002. DOI: https://doi.org/10.1080/02626660209493019

PAREDES-TREJO, F.; BARBOSA, H. A.; SPATAFORA, L. R. Assessment of SM2RAIN-Derived and State-of-the-art satellite rainfall products over Northeastern Brazil. Remote Sensing, v.10, n.7, p.1-29, 2018. DOI: https://doi.org/10.3390/rs10071093

RIEBSAME, W. E.; CHANGNON JUNIOR, S. A.; KARL, T. R.. Drought and natural resources management in the United States: Impacts and implications of the 1987-89 drought. Boulder: Westview Press, 1991.

ROSA, D. B.; SOUSA, R. R.; NASCIMENTO, L. A.; TOLEDO, L. G.; TOPANOTTI, D. Q.; NASCIMENTO, J. A.. A Distribuição Espacial Das Chuvas Na Porção Centro Oeste Do Estado De Mato Grosso-Brasil. Revista Eletrônica da Associação dos Geógrafos Brasileiros, v.1, n.5, p.127-152, 2007.

SANSIGOLO, C. A.. Análise Comparativa do Desempenho dos Índices de Seca de Palmer (PDSI) e da Precipitação Normalizada (SPI) em Piracicaba, SP (1917-2001). Revista Brasileira de Meteorologia, Rio de Janeiro, v.19, n.3, p.237242, 2004. 
SIQUEIRA, B.; NERY, J. T.. Aplicação e análise do índice padronizado de precipitação no circuito das águas paulista. Revista Brasileira de Climatologia, v.16, 2015. DOI: http://dx.doi.org/10.5380/abclima.v16i0.40331

SOUZA, A. P.; MOTA, L. L.; ZAMADEI, T.; MARTIM, C. C.; ALMEIDA, F. T.; PAULINO, J.. Classificação Climática E Balanço Hídrico Climatológico No Estado De Mato Grosso. Nativa, v.1, n.1, p.34-43, 2013. DOI: http://dx.doi.org/10.14583/2318-7670.v01n01a07

TAN, M. L.; TAN, K. C.; CHUA, V. P.; CHAN, N. W.. Evaluation of TRMM product for monitoring drought in the kelantan river basin, Malaysia, Water, v.9, n.57, p.2-15, 2017. DOI: https://doi.org/10.3390/w9010057

TANAKA, A. A.; SOUZA, A. P.; KLAR, A. E.; SILVA, A. C.; GOMES, A. W. A.. Evapotranspiração de referência estimada por modelos simplificados para o Estado do Mato Grosso. Pesquisa Agropecuária Brasileira, v.51, n.2, p.91-104, 2016. DOI: http://dx.doi.org/10.1590/S0100-204X2016000200001

TARIFFA, J. R.; SETTE, D. M.; MADRUGA, L. C. MOREIRA, M. L. C.; ORMOND, G. L.; FILHO, V. D.; SANTOS, J. F.. Atlas

Climatológico de Mato Grosso: Departamento de GeografiaUniversidade Federal de Mato Grosso (UFMT). Rondonópolis: UFMT, 2006.

TUCCI, C. E. M.. Hidrologia: ciência e aplicação. 3 ed. Porto Alegre: ABRH, 2004.
ULIANA, E. M.; REIS, E. F.; SOUZA, P.; SILVA, G. F.; XAVIER, A. C.. Utilização do índice de precipitação padronizado para a região norte do estado do espírito santo. Irriga, v.20, n.3, p.414-428, 2015. DOI: https://doi.org/10.15809/irriga.2015v20n3p414

ULIANA, E. M.; SILVA, D. D.; MOREIRA, M. C.; PEREIRA, S. B. PEREIRA, D. R.. Modelos hidrológicos SAC-SMA e IPH II: calibração e avaliação do desempenho na estimativa de vazões na bacia do rio Piracicaba (MG). Irriga, v.25, n.2, p.202-222, 2020. DOI:

https://doi.org/10.15809/irriga.2020v25n2p202-222

VAN LIEW, M. W.; VEITH, T. L.; BOSCH, D. D.; ARNOLD, J. G. Suitability of SWAT for the Conservation effects assessment project: A comparison on USDA-ARS watersheds. Journal of Hydrologic Engineering, Lincoln, v.12, n.2, p.173-189, 2007. DOI: https://doi.org/10.1061/(ASCE)10840699(2007)12:2(173)

WILHITE, D. A.. Drought as a natural hazard: Concepts and definitions. In: WILHITE, D. A.. Drought: A Global Assessment. New York: Routledge, 2000. p.3-18.

ZHAO, Q.; CHEN, Q.; JIAO, M.; WU, P.; GAO, X.; MA, M.; HONG, Y.. The Temporal-Spatial Characteristics of Drought in the Loess Plateau Using the Remote-Sensed TRMM Precipitation Data from 1998 to 2014. Remote Sensing, v.10, n.838, p.1-20, 2018. DOI:

https://doi.org/10.3390/rs10060838

A CBPC - Companhia Brasileira de Produção Científica (CNPJ: 11.221.422/0001-03) detém os direitos materiais desta publicação. Os direitos referem-se à publicação do trabalho em qualquer parte do mundo, incluindo os direitos às renovaç̃ões, expansões e disseminações da contribuiç̃o, bem como outros direitos subsidiários. Todos os trabalhos publicados eletronicamente poderão posteriormente ser publicados em coletâneas impressas sob coordenação da Sustenere Publishing, da Companhia Brasileira de Produção Científica e seus parceiros autorizados. Os (as) autores (as) preservam os direitos autorais, mas não têm permissão para a publicação da contribuição em outro meio, impresso ou digital, em português ou em tradução. 